\title{
The Postsynaptic Density: There Is More than Meets the Eye
}

\author{
Ayse Dosemeci ${ }^{1 *}$, Richard J. Weinberg ${ }^{2}$, Thomas S. Reese ${ }^{1}$ and Jung-Hwa Tao-Cheng ${ }^{3}$ \\ ${ }^{1}$ Laboratory of Neurobiology, National Institute of Neurological Disorders and Stroke (NINDS), National Institutes \\ of Health (NIH), Bethesda, MD, USA, ${ }^{2}$ Department of Cell Biology and Physiology, University of North Carolina, Chapel Hill, \\ Chapel Hill, NC, USA, ${ }^{3}$ Electron Microscopy Facility, National Institute of Neurological Disorders and Stroke (NINDS), \\ National Institutes of Health (NIH), Bethesda, MD, USA
}

The postsynaptic density (PSD), apparent in electron micrographs as a dense lamina just beneath the postsynaptic membrane, includes a deeper layer, the "pallium", containing a scaffold of Shank and Homer proteins. Though poorly defined in traditionally prepared thin-section electron micrographs, the pallium becomes denser and more conspicuous during intense synaptic activity, due to the reversible addition of CaMKII and other proteins. In this Perspective article, we review the significance of CaMKII-mediated recruitment of proteins to the pallium with respect to both the trafficking of receptors and the remodeling of spine shape that follow synaptic stimulation. We suggest that the level and duration of CaMKII translocation and activation in the pallium will shape activity-induced changes in the spine.

Keywords: postsynaptic density, PSD, pallium, electron microscopy, EM, Shank, Homer, CaMKII

OPEN ACCESS

Edited by:

George Augustine, Nanyang Technologicial University (NTU), Singapore

Reviewed by:

Eric Hanse,

University of Gothenburg, Sweden Anne McKinney,

McGill University, Canada

*Correspondence: Ayse Dosemeci dosemeca@mail.nih.gov

Received: 17 May 2016 Accepted: 25 July 2016 Published: 19 August 2016

Citation:

Dosemeci A, Weinberg RJ, Reese TS and Tao-Cheng J-H (2016) The Postsynaptic Density: There Is More than Meets the Eye. Front. Synaptic Neurosci. 8:23. doi: 10.3389/fnsyn.2016.00023
Excitatory glutamatergic synapses examined with the electron microscope typically display a pronounced postsynaptic density (PSD), which appears in conventional electron micrographs as an approximately $30 \mathrm{~nm}$ thick electron-dense structure applied to the postsynaptic membrane (Palay, 1956). The large majority of excitatory synapses in the vertebrate CNS release glutamate as neurotransmitter. Ionotropic glutamate receptors concentrate at the PSD, where specialized molecules anchor them and regulate their trafficking; modulation of their expression and trafficking plays a key role in synaptic plasticity. Decades of research show that much of the story of this modulation resides in the PSD.

Using special stains, Valtschanoff and Weinberg (2001) uncovered a distinct layer $\sim 50 \mathrm{~nm}$ thick immediately adjacent and deep to the PSD. Though previously reported, this "subsynaptic web" (DeRobertis, 1964) had been largely ignored for decades, because it is difficult to distinguish from the rest of the spine cytoplasm after standard histological procedures. Extending this work, Petralia et al. (2005) reported high levels of immunoEM label for both Shank and Homer in the same region, which was referred to as the "subjacent" area.

The subsynaptic web becomes more prominent after short bouts of stimulation, reflecting the reversible incorporation of additional proteins. Because this part of the PSD comprises different proteins and displays a more labile organization than the "core" layer lining the synaptic membrane, it merits a distinct name; we propose to designate this separate structural and functional layer of the PSD as its pallium or mantle (Figure 1). Here we present a perspective on PSD structure that highlights potential functions carried out in the pallium.

\section{CORE LAYER OF THE PSD}

Though highly variable, PSDs in the rodent forebrain contain on average 300-400 copies of PSD-95 and related membrane-associated guanylate kinase (MAGUK) molecules (Chen et al., 2005; 


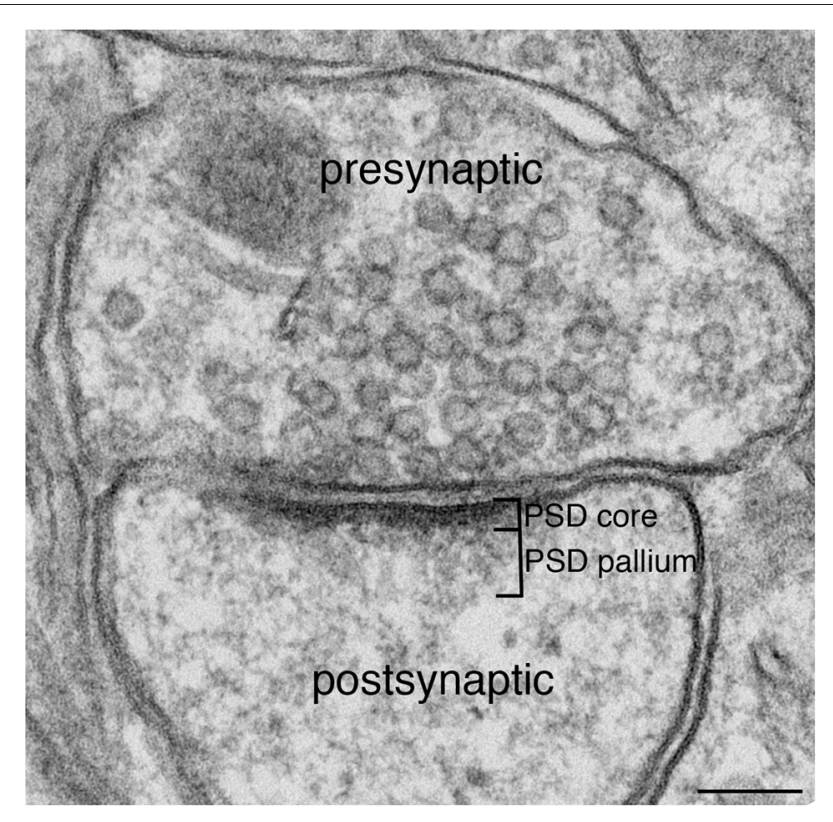

FIGURE 1 | Excitatory synapse. Excitatory glutamatergic synapses typically display a prominent electron-dense zone juxtaposed to the postsynaptic membrane, traditionally known as the postsynaptic density (PSD). Extending deeper from the PSD core is a specialized region we call the PSD pallium. Scale bar: $0.1 \mu \mathrm{m}$.

Sugiyama et al., 2005). PSD-95 is localized in the core layer of the PSD. Comparing immunogold labeling with antibodies against its $\mathrm{N}$ - and C-termini shows that PSD-95 is oriented with its $\mathrm{N}$-terminus near the plane of the postsynaptic membrane and its C-terminus deep in the spine (Chen et al., 2008). Electron microscopic tomography reveals vertical filaments extending from the postsynaptic membrane in numbers and dimensions expected for PSD-95 (Chen et al., 2008), and these filaments are depleted by acute knockdown of PSD-95 (Chen et al., 2011). Thus, PSD-95-containing filaments oriented vertically to the postsynaptic membrane represent a major structural component of the core layer (Chen et al., 2008). This close apposition to the membrane puts PSD95 in a position to bind neurotransmitter receptors. Indeed, there is substantial evidence indicating the association of PSD-95 with both NMDA and AMPA types of glutamate receptors.

EM tomography also reveals filaments of different lengths, oriented parallel to the postsynaptic membrane, that appear to contact the deep (C-terminal) ends of PSD-95 filaments (Chen et al., 2008). The dimensions of one frequently-encountered type of horizontal filament corresponds to that expected for guanylate kinase-associated protein (GKAP), consistent with biochemical work demonstrating that GKAP can bind PSD95 near its C-terminal (Kim et al., 1997). GKAP is in the PSD at a ratio of approximately one GKAP for two PSD95s (Lowenthal et al., 2015) so a layer of GKAP cross-linked with PSD-95 could provide an interface between the core layer and the pallium of the PSD. Like PSD-95 (Yang et al., 2011), GKAP stays put during brief stimulation (Tao-Cheng et al.,

\section{BOX 1 | Major scaffold proteins in the PSD.}

Names used in the present article are listed along with synonyms.

MAGUKs (membrane-associated guanylate kinases).

The most abundant MAGUK at the mammalian PSD is PSD-95 (DLG4, SAP90); others include SAP97 (DLG1), PSD-93 (DLG2, Chapsyn-110), and SAP102 (DLG3).

GKAP (guanylate kinase-associated protein) also called SAPAP (SAP90/PSD95-associated protein), or disks large-associated protein.

Shank (SH3 and multiple ankyrin repeat domains protein) also called ProSAP (Proline-rich synapse-associated protein), Synamon and CortBP (Cortactin binding protein).

Homer also called Vesl, Cupidin, and PSD-Zip45.

2015). Thus, the dense matrix of PSD-95/MAGUK filaments capped by GKAPs provides a stable platform for the subjacent pallium.

\section{PALLIAL LAYER OF THE PSD}

Early biochemical analyses indicated that the PSD complex contains several types of scaffolding molecules in addition to MAGUKs and GKAPs (Box 1). Two-hybrid and co-immunoprecipitation experiments led to the identification of the Shank/ProSAP family of proteins, which bind to GKAPs (Boeckers et al., 1999a,b; Naisbitt et al., 1999). Similar twohybrid studies identified the Homer family of proteins as binding partners for Shanks and the two types of proteins were shown to co-immunoprecipitate from brain extracts (Tu et al., 1999). Both Shank and Homer family proteins are highly enriched in PSD fractions (Xiao et al., 1998; Naisbitt et al., 1999) and co-purify as large complexes, with PSD-95, GKAP and other PSD constituents (Collins et al., 2006; Dosemeci et al., 2007).

While biochemical studies identify Shank and Homer as part of the PSD complex, immunoEM studies show that label for these proteins concentrates outside the electron-dense material conventionally defined as the PSD (Figure 2). Although the reported distributions of label for these proteins differ somewhat between groups, immunoEM studies typically find that much of the postsynaptic immunogold label for both Shank and Homer lie in a layer immediately below the PSD core (Tu et al., 1999; Valtschanoff and Weinberg, 2001; Petralia et al., 2005; Rostaing et al., 2006; Tao-Cheng et al., 2010, 2014a).

These data imply that a network containing Shanks and Homer extends the PSD scaffold well beyond the classically recognized electron-dense material. This second layer, which we term the pallium, is likely pegged to the PSD core through GKAPs, which can simultaneously associate with PSD-95 and Shanks. In vitro studies report that Shanks can associate with each other through their SAM domains to form sheet-like structures (Baron et al., 2006) and Homers can polymerize into tetramers that can cross-link Shanks (Hayashi et al., 2009). Furthermore, purified C-terminal Shank and Homer form a mesh-like matrix when mixed together (Hayashi et al., 2009). Thus, Shank and Homer proteins in the pallium are likely to form an extended scaffold. The side of this scaffold facing the cleft is continuous 
A

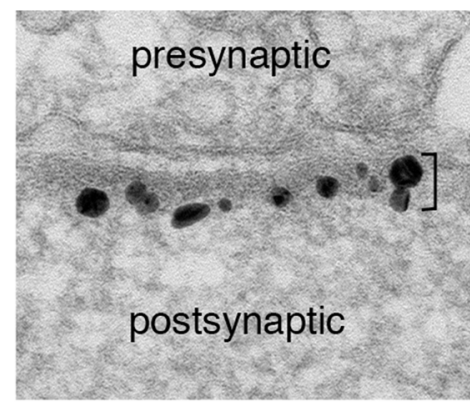

PSD 95

B

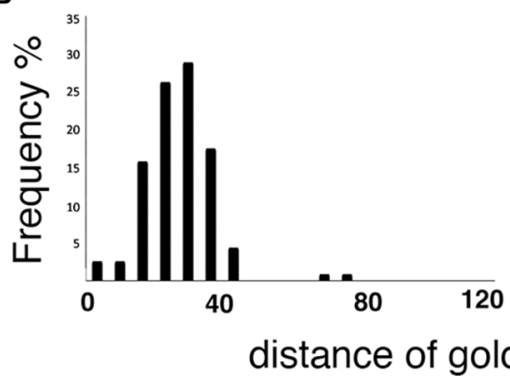

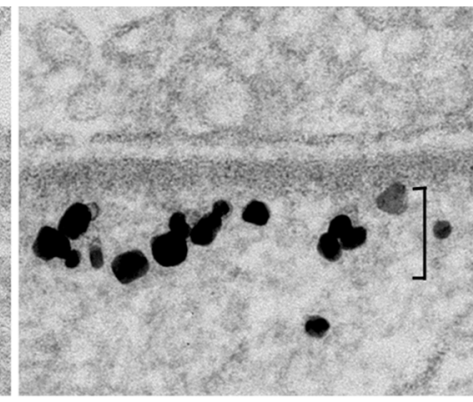

Shank

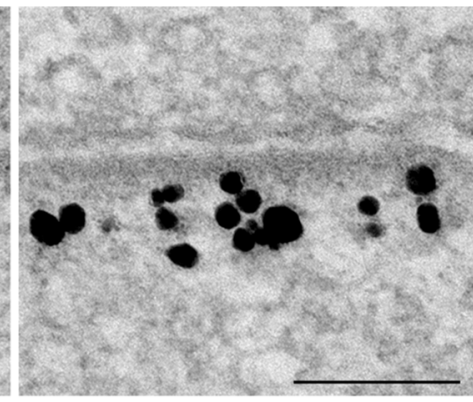

Homer

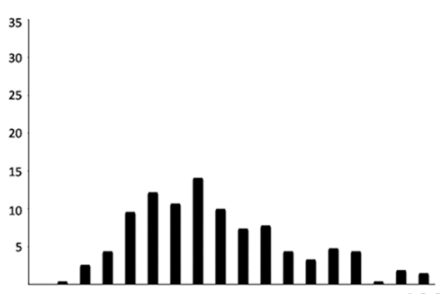

40

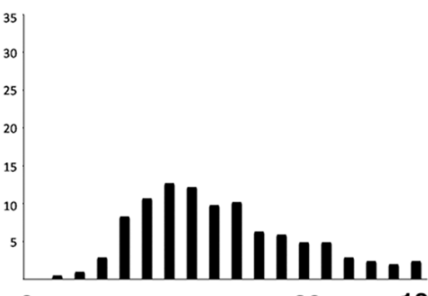

120

FIGURE 2 | Label for two PSD scaffold proteins, Shank and Homer, lies mostly outside the PSD core. (A) Electron micrographs of asymmetric synapses in cultured hippocampal neurons immunogold labeled with antibodies for three major PSD scaffold proteins. Silver-enhanced gold label appears as black particles of heterogeneous size (EM in middle panel from Tao-Cheng et al., 2015). The PSD core with PSD-95 label and the PSD pallium with Shank and Homer labels are marked by brackets on the left and middle panels respectively. Scale bar: $0.1 \mu \mathrm{m}$. (B) Frequency histograms depicting the distribution of label at the postsynaptic compartment in a typical experiment. While label for PSD-95 is within the electron-dense material, label for Shank and Homer is concentrated in a deeper region we designate as the pallium of the PSD.

with the PSD core, whereas the cytoplasmic side, characterized by an extremely rough surface (Petersen et al., 2003) merges imperceptibly into the central zone of the spine head.

\section{ACTIVITY-INDUCED CHANGES AT THE PALLIUM}

\section{The Pallium Becomes More Electron- Dense as CaMKII Accumulates}

Thin-section EM of cultured hippocampal neurons reveals increased electron density at the pallium under excitatory conditions (Figure 3: top panels), a phenomenon we have described as PSD "thickening" (Dosemeci et al., 2001). The increase in electron density likely reflects the addition of proteins, and immunoEM shows significant increase in CaMKII immunolabel within the PSD complex under excitatory conditions (Figure 3: bottom panels, Dosemeci et al., 2001). Similar morphological changes at the PSD, accompanied by the accumulation of CaMKII and other proteins, also occur under the excitotoxic conditions promoted by ischemia (Suzuki et al., 1994; Hu et al., 1998; Martone et al., 1999). The duration of the morphological and compositional changes at the PSD appears to vary according to the type of stimulation; importantly, more sustained modification is observed following an LTP-inducing protocol (Otmakhov et al., 2004).

\section{Role of CaMKII as a Kinase}

CaMKII, a $\mathrm{Ca}^{2+}$-regulated serine-threonine protein kinase, is present at very high concentrations in neurons, with a relative abundance reaching $\sim 1-2 \%$ of total protein in the cerebral cortex and hippocampus (Erondu and Kennedy, 1985). Within neurons, CaMKII is especially prominent in the PSD pallium (Petralia et al., 2005; Ding et al., 2013). Activation of CaMKII plays a pivotal role in certain types of synaptic modification, most notably NMDA-dependent LTP (reviews: Lisman et al., 2012; Shonesy et al., 2014).

The CaMKII holoenzyme is a dodecamer made of individually active subunits, with $\alpha$ - and $\beta$-isoforms prevalent in brain. Subunits within a single holoenzyme can phosphorylate one another, a process called autophosphorylation. Upon autophosphorylation in the presence of $\mathrm{Ca}^{2+}$, CaMKII becomes autonomous, allowing enzymatic activity to persist beyond the duration of $\mathrm{Ca}^{2+}$ elevation (reviews: Hell, 2014; Shonesy et al., 2014). Autonomous CaMKII can remain at the PSD pallium after the cessation of excitatory stimuli (Dosemeci et al., 2002; Otmakhov et al., 2004). Continued co-localization of autonomous CaMKII with its substrates at the pallium would counteract dephosphorylation by phosphatases and thus help maintain phosphorylation of CaMKII substrates.

The accumulation and activation of CaMKII at the pallium triggers further changes at the PSD. Some of these have been studied in depth, such as the incorporation of AMPA receptors 


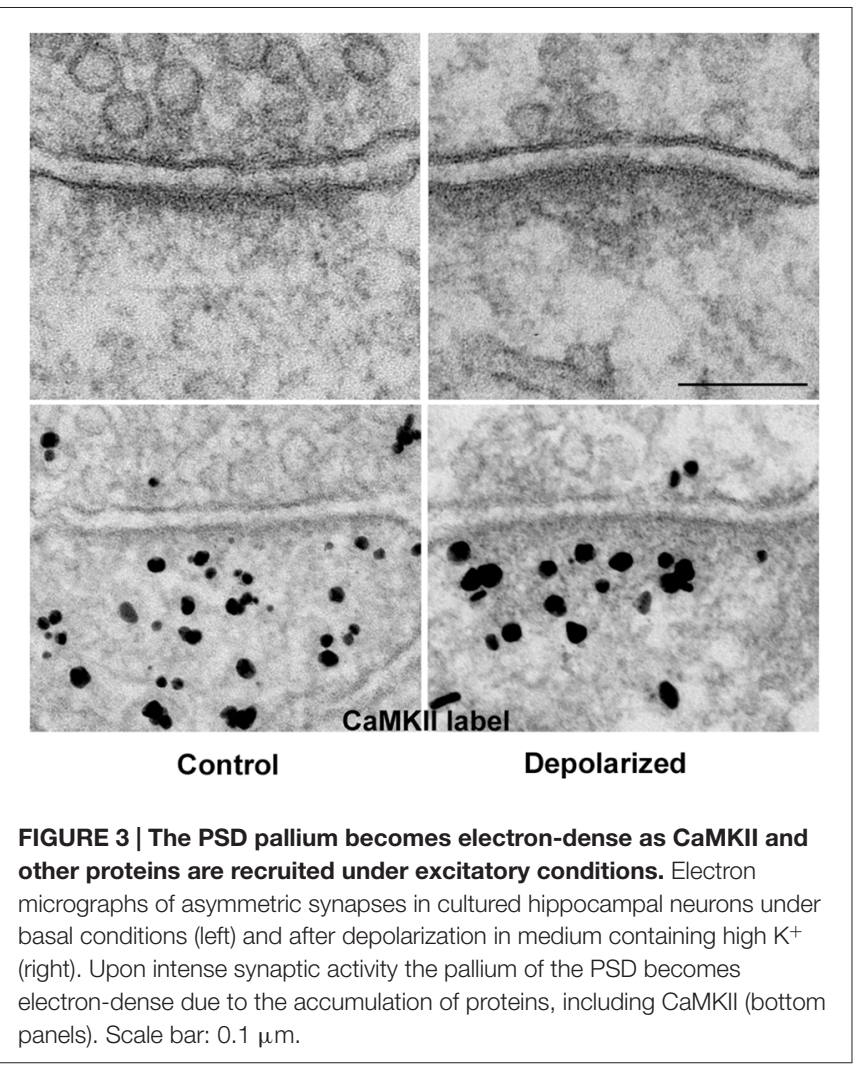

into the postsynaptic membrane (review: Lisman et al., 2012). ImmunoEM studies also reveal activity-induced increases in the levels of a number of proteins at the pallium, including SynGAP, AIDA-1 and Shanks, in parallel with CaMKII. Importantly, the redistribution of all these PSD components is blocked by the application of a specific CaMKII inhibitor (Yang et al., 2013; TaoCheng et al., 2014b; Dosemeci et al., 2016), indicating that the activation of CaMKII is required for the redistribution of these PSD components.

\section{Role of CaMKII as a Dynamic Structural Element}

In addition to its function as a protein kinase, accumulating evidence suggests that CaMKII in the pallium may act as a scaffold. Bingol et al. (2010) show that activity-induced translocation of autophosphorylated $\alpha$-CaMKII, but not its kinase activity, is responsible for the translocation of proteasomes into synapses. Similarly, activity-induced CaMKII translocation is responsible for parallel accumulation of the deubiquitinase CYLD, specific for K63-linked polyubiquitins, to the PSD (Thein et al., 2014). Since K63linked polyubiquitination inhibits interaction of proteins with proteasomes (Nathan et al., 2013) CYLD activity is expected to facilitate protein binding to proteasomes. Thus, CaMKII accumulated at the pallium appears to constitute a structural platform that brings together components that may work synergistically to promote local degradation of proteins.

Activity-induced translocation of CaMKII also may mediate modifications in spine morphology. CaMKII holoenzymes at the spine cytoplasm serve as actin bundling elements (Okamoto et al., 2007) and dissociation of CaMKII from F-actin upon autophosphorylation destabilizes the actin cytoskeleton (Kim et al., 2015). Thus the translocation of CaMKII from the actin network to the PSD pallium can trigger synchronized changes at the PSD and actin cytoskeleton.

\section{SynGAP Levels Decrease in the PSD Core and Increase in the Pallium}

SynGAP is an enzymatic regulator of Ras, a small GTPase. However, as one of the most abundant proteins at the PSD (at levels exceeding even those of PSD-95, its binding partner at the PSD core), SynGAP is likely to also play a non-enzymatic role. ImmunoEM shows that SynGAP label is concentrated at the PSD core (Yang et al., 2011, 2013). After strong depolarization, labeling for SynGAP significantly decreases at the PSD core while increasing at the pallium (Yang et al., 2011, 2013). Under the same conditions, label for PSD-95 does not change its localization at the PSD (Yang et al., 2011), implying dissociation of the two molecules. Further studies indicate that the release of SynGAP from PSD-95 and its exit from the PSD core require CaMKIImediated phosphorylation (Yang et al., 2013; Araki et al., 2015).

NMDA- or high $\mathrm{K}^{+}$-induced changes in the distribution of SynGAP are reversed within $30 \mathrm{~min}$ after cessation of the excitatory conditions. In contrast, a stimulation protocol (glycine in the absence of $\mathrm{Mg}^{2+}$ ) that leads to sustained increases in synaptic efficacy (chem-LTP) also leads to a sustained exclusion of SynGAP from the spine (Araki et al., 2015) indicating a correlation between LTP and the removal of SynGAP. It appears likely that a wide range of excitatory stimuli promotes translocation of SynGAP out of the PSD core, but that only under LTP-promoting conditions is the molecule removed on a long-term basis. The extent and maintenance of SynGAP phosphorylation at the pallium may determine its subsequent movement. In this regard, it is interesting that SynGAP is most efficiently phosphorylated by the autonomous form of CaMKII in the absence of $\mathrm{Ca}^{2+}$ (Dosemeci and Jaffe, 2010).

What might be the functional implications of activity-induced redistribution of SynGAP at the PSD? Both SynGAP and members of the transmembrane AMPA receptor regulatory protein family (TARPs) can bind to the same PDZ domains on PSD-95 (Kim et al., 1998; Schnell et al., 2002; Dakoji et al., 2003) and thus may compete for association with PSD-95. Considering its high abundance in the PSD, SynGAP is likely to block TARP binding to PDZ domains on PSD-95 under basal conditions. Thus, the removal of SynGAP during activity may be a prerequisite for the anchoring of AMPA receptors to the PSD, explaining the well-documented role of SynGAP as an inhibitor of AMPA receptor insertion (Rumbaugh et al., 2006). This function may account for the remarkable abundance of SynGAP at the PSD.

\section{AIDA-1 Levels Decrease in the PSD Core and Increase in the Pallium}

Quantitative mass spectrometric analysis of PSD fractions reveals that Amyloid- $\beta$ protein precursor Intracellular Domain- 
Associated 1 (AIDA-1, also known as EB-1 and ANKS1B) is a major component of the PSD complex, with a PSD-95/AIDA1/GKAP stoichiometry of 2:1:1 (Lowenthal et al., 2015). Like SynGAP, AIDA-1 binds directly to PSD-95 at the same domains that bind TARPs (Jordan et al., 2007) and therefore could also interfere with the anchoring of AMPA receptors. By immunoEM, the AIDA-1 label is mostly located within the PSD core at rest (Jacob et al., 2010; Dosemeci et al., 2015). Under excitatory conditions AIDA-1 label at the PSD core is significantly reduced with a concomitant increase at the pallium (Dosemeci et al., 2015). The reversible CaMKII-mediated redistribution of AIDA-1 at the PSD under excitatory conditions (Dosemeci et al., 2016) parallels that of SynGAP. We speculate that different regulatory mechanisms may trigger dissociation of SynGAP and AIDA-1 from PSD-95.

\section{Shank Levels Increase in the Pallium}

Shanks, a protein scaffold family concentrated in the PSD, are encoded by three genes, Shank1, Shank2 and Shank3, each giving rise to multiple splice variants. Shank mutations have been linked to autism and other neurodevelopmental/neuropsychiatric disorders (reviews: Grabrucker et al., 2011; Sala et al., 2015). All Shank isoforms have a similar molecular organization, with specialized domains that can associate with GKAPs, Homers, and other Shanks. Analysis of immunoEM data suggests that Shanks at the PSD are organized into a proximal pool, close enough to the interface between the core and pallium to associate with GKAP, and a distal (deeper) pool that may be stabilized through association with Homers and/or with other Shanks. Under excitatory conditions, Shanks accumulate preferentially in the distal pool (Tao-Cheng et al., 2015).

Shanks promote maturation of dendritic spines and the enlargement of spine heads (Sala et al., 2001), although the precise mechanism remains unclear. Considering that changes in spine shape and size involve reorganization of the actin cytoskeleton, it is likely that Shanks regulate spine morphology through interaction with actin. Indeed, Shanks associate with three actin-regulating proteins, Insulin Receptor Substrate Protein 53 (IRSp53), Abp1 and cortactin.

The actin binding protein IRSp53 (also called BAIAP2; see review by Kang et al., 2016) is a major PSD component, with a molar ratio of IRSp53 to PSD-95 of 1:4 (Lowenthal et al., 2015). IRSp53 is located between layers containing Shank and PSD-95, relatively close to the postsynaptic membrane (Burette et al., 2014), where it may function as a linker between the actin cytoskeleton and the plasma membrane (Scita et al., 2008; Burette et al., 2014). Indeed, IRSp53 contains an actin-binding BAR-like domain that can induce changes in membrane curvature (Zhao et al., 2011) and thus may be involved in activity-induced changes in the curvature of the synapse (Burette et al., 2014).

Abp1, which can associate simultaneously with actin and Shanks, may link the actin cytoskeleton to the PSD (Qualmann et al., 2004). Abp1 preferentially interacts with dynamic rather than static F-actin structures (Kessels et al., 2000) and more Abp1 becomes incorporated into Shank-positive synapses during activity (Qualmann et al., 2004). Overexpression of Abp1
TABLE 1 | Redistribution of postsynaptic density (PSD) components during activity.

\begin{tabular}{llllll}
\hline & CaMKII & SynGAP & AIDA-1 & Shank & CYLD \\
\hline Core & & $\begin{array}{l}\text { decrease } \\
\text { increase }\end{array}$ & $\begin{array}{l}\text { decrease } \\
\text { increase }\end{array}$ & increase & increase
\end{tabular}

Changes observed in immunogold label density for selected proteins following treatment of cultured hippocampal neurons with media containing NMDA or high $\mathrm{K}^{+}$.

increases the density of mushroom-shaped spines; importantly, its association with Shank is necessary for Abp1-mediated regulation of spine morphology (Haeckel et al., 2008).

Another protein that associates with both Shanks and actin is cortactin, although immunoEM shows that cortactin concentrates mainly at the central region of the spine (Racz and Weinberg, 2004). In contrast to Abp1, cortactin exits the spines during activity (Hering and Sheng, 2003). While Abp1 overexpression increases mushroom spine density (Haeckel et al., 2008), cortactin overexpression causes elongation of spines (Hering and Sheng, 2003).

The above considerations lead us to suggest a model for activity-induced modification of spine morphology: under basal conditions, cytosolic Shanks within the spine are pegged to the actin cytoskeleton through cortactin and held outside of the pallium. During activity, CaMKII-mediated phosphorylation releases Shanks from cortactin. The Shank thus released accumulates at the pallium, while cortactin exits the spine. Shank accumulated at the pallium could then recruit Abp1 to promote remodeling of the actin cytoskeleton around the pallium.

\section{ACTIN AND THE PSD}

There are several reports that filaments of F-actin, the primary cytoskeletal component of the spine, contact the PSD (Gulley and Reese, 1981; Landis and Reese, 1983; Fifková, 1985), especially at its periphery (Burette et al., 2012), though these contacts are likely highly variable, considering the dynamic nature of F-actin. While the molecular basis of the attachment of actin filaments to the PSD remains uncertain, a number of PSD molecules are plausible candidates. As discussed above, two Shank binding proteins, IRSp53 and Abp1, may provide a link between the PSD and the actin cytoskeleton.

Yet another point of interaction of actin with the PSD may be through the ubiquitous actin binding protein $\alpha$-actinin. In vitro assays show that $\alpha$-actinin can form a ternary complex with the PSD proteins, densin and CaMKII (Walikonis et al., 2001). Loss of $\alpha$-actinin-2 in rat hippocampal neurons creates an increased density of immature, filopodia-like protrusions that fail to mature into a mushroom-shaped spine during development (Hodges et al., 2014).

\section{CONCLUSION}

The pallium is an extension of the electron-dense PSD core, demarcated by strong immunolabeling for two PSD 
scaffold proteins, Shank and Homer. This specialized zone, sandwiched between the electron-dense PSD core and the actin "spinoskeleton", is highly dynamic, exhibiting striking changes during synaptic activity. Under conditions of strong excitation, the pallium becomes electron-dense, with the addition of CaMKII and several other proteins (Table 1). Activation and/or translocation of CaMKII is necessary for the recruitment of other components to the pallium.

The pallium can be viewed as a hub where several proteins converge during activity. Accumulating evidence on the movements of individual proteins suggests a mechanism for concerted insertion of receptors to the PSD and re-organization of the actin spinoskeleton, both mediated by CaMKII. Upon synaptic stimulation, translocation and activation of CaMKII cause SynGAP and AIDA-1 to move out of the PSD core and accumulate at the pallium (Yang et al., 2013; Dosemeci et al., 2015, 2016). We propose that the movement of SynGAP (and perhaps also of AIDA-1) vacates "slots" on PSD-95, providing a window of opportunity for the insertion of AMPA receptors. Simultaneous CaMKII-mediated accumulation of Shanks at the pallium (Tao-Cheng et al., $2014 b$ ), on the other hand, may enable actin reorganization around the PSD. CaMKII also acts as a dynamic structural element whose activity-induced translocation changes the molecular organization within the spine. Dissociation of CaMKII from F-actin causes destabilization and reorganization

\section{REFERENCES}

Araki, Y., Zeng, M., Zhang, M., and Huganir, R. L. (2015). Rapid dispersion of SynGAP from synaptic spines triggers AMPA receptor insertion and spine enlargement during LTP. Neuron 85, 173-189. doi: 10.1016/j.neuron.2014. 12.023

Baron, M. K., Boeckers, T. M., Vaida, B., Faham, S., Gingery, M., Sawaya, M. R., et al. (2006). An architectural framework that may lie at the core of the postsynaptic density. Science 311, 531-535. doi: 10.1126/science.1118995

Bingol, B., Wang, C. F., Arnott, D., Cheng, D., Peng, J., and Sheng, M. (2010). Autophosphorylated CaMKII $\alpha$ acts as a scaffold to recruit proteasomes to dendritic spines. Cell 140, 567-578. doi: 10.1016/j.cell.2010.01.024

Boeckers, T. M., Kreutz, M. R., Winter, C., Zuschratter, W., Smalla, K. H., Sanmarti-Vila, L., et al. (1999a). Proline-rich synapse-associated protein$1 /$ cortactin binding protein 1 (ProSAP1/CortBP1) is a PDZ-domain protein highly enriched in the postsynaptic density. J. Neurosci. 19, 6506-6518.

Boeckers, T. M., Winter, C., Smalla, K. H., Kreutz, M. R., Bockmann, J., Seidenbecher, C., et al. (1999b). Proline-rich synapse-associated proteins ProSAP1 and ProSAP2 interact with synaptic proteins of the SAPAP/GKAP family. Biochem. Biophys. Res. Commun. 264, 247-252. doi: 10.1006/bbrc. 1999.1489

Burette, A. C., Lesperance, T., Crum, J., Martone, M., Volkmann, N., Ellisman, M. H., et al. (2012). Electron tomographic analysis of synaptic ultrastructure. J. Comp. Neurol. 520, 2697-2711. doi: 10.1002/cne.23067

Burette, A. C., Park, H., and Weinberg, R. J. (2014). Postsynaptic distribution of IRSp53 in spiny excitatory and inhibitory neurons. J. Comp. Neurol. 522, 2164-2178. doi: 10.1002/cne.23526

Chen, X., Nelson, C. D., Li, X., Winters, C. A., Azzam, R., Sousa, A. A., et al. (2011). PSD-95 is required to sustain the molecular organization of the postsynaptic density. J. Neurosci. 31, 6329-6338. doi: 10.1523/JNEUROSCI.5968-10.2011

Chen, X., Vinade, L., Leapman, R. D., Petersen, J. D., Nakagawa, T., Phillips, T. M., et al. (2005). Mass of the postsynaptic density and enumeration of three key molecules. Proc. Natl. Acad. Sci. U S A 102, 11551-11556. doi: 10.1073/pnas. 0505359102 of the actin cytoskeleton (Kim et al., 2015). Subsequent accumulation of CaMKII at the pallium docks elements that regulate protein turnover (Bingol et al., 2010; Thein et al., 2014).

In summary, activation of CaMKII and its translocation to the pallium under excitatory conditions trigger a chain of events poised to elicit profound effects on the organization of the PSD complex and of the actin cytoskeleton that could synchronize receptor trafficking with changes in spine morphology. We speculate that the degree and duration of CaMKII accumulation and activity at the pallium, promoted by different types of excitatory stimuli, may determine the type and level of synaptic modification.

\section{AUTHOR CONTRIBUTIONS}

All authors contributed to the writing of the manuscript.

\section{ACKNOWLEDGMENTS}

We would like to thank Dr. John Lisman for stimulating discussions and for a critical reading of the manuscript. This work was supported by the Intramural Research Program of the National Institute of Neurological Disorders and Stroke (NINDS)/National Institutes of Health (NIH) and NINDS/NIH NS 039444 grant to RJW.

Chen, X., Winters, C., Azzam, R., Li, X., Galbraith, J. A., Leapman, R. D. et al. (2008). Organization of the core structure of the postsynaptic density. Proc. Natl. Acad. Sci. U S A 105, 4453-4458. doi: 10.1073/pnas.08008 97105

Collins, M. O., Husi, H., Yu, L., Brandon, J. M., Anderson, C. N., Blackstock, W. P., et al. (2006). Molecular characterization and comparison of the components and multiprotein complexes in the postsynaptic proteome. J. Neurochem. 97, 16-23. doi: 10.1111/j.1471-4159.2005.03507.x

Dakoji, S., Tomita, S., Karimzadegan, S., Nicoll, R. A., and Bredt, D. S. (2003). Interaction of transmembrane AMPA receptor regulatory proteins with multiple membrane associated guanylate kinases. Neuropharmacology 45, 849-856. doi: 10.1016/s0028-3908(03)00267-3

DeRobertis, E. D. P. (1964). "International series of monographs on pure and applied biology. Division, modern trends in physiological sciences," in Histophysiology of Synapses and Neurosecretion (Vol. 20), eds P. Alexander and Z. M. Bacq (Oxford: Pergamon Press), 34-42.

Ding, J. D., Kennedy, M. B., and Weinberg, R. J. (2013). Subcellular organization of camkii in rat hippocampal pyramidal neurons. J. Comp. Neurol. 521, 3570-3583. doi: 10.1002/cne.23372

Dosemeci, A., and Jaffe, H. (2010). Regulation of phosphorylation at the postsynaptic density during different activity states of $\mathrm{Ca}^{2+} /$ calmodulindependent protein kinase II. Biochem. Biophys. Res. Commun. 391, 78-84. doi: 10.1016/j.bbrc.2009.10.167

Dosemeci, A., Makusky, A. J., Jankowska-Stephens, E., Yang, X., Slotta, D. J., and Markey, S. P. (2007). Composition of the synaptic PSD-95 complex. Mol. Cell. Proteomics 6, 1749-1760. doi: 10.1074/mcp.M700040MCP200

Dosemeci, A., Tao-Cheng, J. H., Vinade, L., Winters, C. A., Pozzo-Miller, L., and Reese, T. S. (2001). Glutamate-induced transient modification of the postsynaptic density. Proc. Natl. Acad. Sci. U S A 98, 10428-10432. doi: 10. 1073/pnas.181336998

Dosemeci, A., Toy, D., Burch, A., Bayer, K. U., and Tao-Cheng, J. H. (2016). CaMKII-mediated displacement of AIDA-1 out of the postsynaptic density core. FEBS Lett. doi: 10.1002/1873-3468.12334 [Epub ahead of print]. 
Dosemeci, A., Toy, D., Reese, T. S., and Tao-Cheng, J. H. (2015). AIDA-1 moves out of the postsynaptic density core under excitatory conditions. PLoS One 10:e0137216. doi: 10.1371/journal.pone.0137216

Dosemeci, A., Vinade, L., Winters, C. A., Reese, T. S., and Tao-Cheng, J. H. (2002). Inhibition of phosphatase activity prolongs NMDA-induced modification of the postsynaptic density. J. Neurocytol. 31, 605-612. doi: 10. 1023/A:1025735410738

Erondu, N. E., and Kennedy, M. B. (1985). Regional distribution of type II $\mathrm{Ca}^{2+} /$ calmodulin-dependent protein kinase in rat brain. J. Neurosci. 5, 3270-3277.

Fifková, E. (1985). Actin in the nervous system. Brain Res. 356, 187-215. doi: 10. 1016/0165-0173(85)90012-8

Grabrucker, A. M., Schmeisser, M. J., Schoen, M., and Boeckers, T. M. (2011). Postsynaptic ProSAP/Shank scaffolds in the cross-hair of synaptopathies. Trends Cell Biol. 21, 594-603. doi: 10.1016/j.tcb.2011.07.003

Gulley, R. L., and Reese, T. S. (1981). Cytoskeletal organization at the postsynaptic complex. J. Cell Biol. 91, 298-302. doi: 10.1083/jcb.91.1.298

Haeckel, A., Ahuja, R., Gundelfinger, E. D., Qualmann, B., and Kessels, M. M. (2008). The actin-binding protein Abpl controls dendritic spine morphology and is important for spine head and synapse formation. J. Neurosci. 28, 10031-10044. doi: 10.1523/jneurosci.0336-08.2008

Hayashi, M. K., Tang, C., Verpelli, C., Narayanan, R., Stearns, M. H., Xu, R. M., et al. (2009). The postsynaptic density proteins Homer and Shank form a polymeric network structure. Cell 137, 159-171. doi: 10.1016/j.cell.2009.01.050

Hell, J. W. (2014). CaMKII: claiming center stage in postsynaptic function and organization. Neuron 81, 249-265. doi: 10.1016/j.neuron.2013.12.024

Hering, H., and Sheng, M. (2003). Activity-dependent redistribution and essential role of cortactin in dendritic spine morphogenesis. J. Neurosci. 23, 11759-11769.

Hodges, J. L., Vilchez, S. M., Asmussen, H., Whitmore, L. A., and Horwitz, A. R. (2014). $\alpha$-Actinin-2 mediates spine morphology and assembly of the postsynaptic density in hippocampal neurons. PLoS One 9:e101770. doi: 10. 1371/journal.pone.0101770

Hu, B. R., Park, M., Martone, M. E., Fischer, W. H., Ellisman, M. H., and Zivin, J. A. (1998). Assembly of proteins to postsynaptic densities after transient cerebral ischemia. J. Neurosci. 18, 625-633.

Jacob, A. L., Jordan, B. A., and Weinberg, R. J. (2010). Organization of amyloidbeta protein precursor intracellular domain-associated protein-1 in the rat brain. J. Comp. Neurol. 518, 3221-3236. doi: 10.1002/cne.22394

Jordan, B. A., Fernholz, B. D., Khatri, L., and Ziff, E. B. (2007). Activity-dependent AIDA-1 nuclear signaling regulates nucleolar numbers and protein synthesis in neurons. Nat. Neurosci. 10, 427-435. doi: 10.1038/nn1867

Kang, J., Park, H., and Kim, E. (2016). IRSp53/BAIAP2 in dendritic spine development, NMDA receptor regulation and psychiatric disorders. Neuropharmacology 100, 27-39. doi: 10.1016/j.neuropharm.2015.06.019

Kessels, M. M., Engqvist-Goldstein, A. E., and Drubin, D. G. (2000). Association of mouse actin-binding protein 1 (mAbp1/SH3P7), an Src kinase target, with dynamic regions of the cortical actin cytoskeleton in response to Rac1 activation. Mol. Biol. Cell 11, 393-412. doi: 10.1091/mbc.11.1.393

Kim, K., Lakhanpal, G., Lu, H. E., Khan, M., Suzuki, A., Hayashi, M. K., et al. (2015). A temporary gating of actin remodeling during synaptic plasticity consists of the interplay between the kinase and structural functions of CaMKII. Neuron 87, 813-826. doi: 10.1016/j.neuron.2015.07.023

Kim, J. H., Liao, D., Lau, L. F., and Huganir, R. L. (1998). SynGAP: a synaptic RasGAP that associates with the PSD-95/SAP90 protein family. Neuron 20, 683-691. doi: 10.1016/s0896-6273(00)81008-9

Kim, E., Naisbitt, S., Hsueh, Y. P., Rao, A., Rothschild, A., Craig, A. M., et al. (1997). GKAP, a novel synaptic protein that interacts with the guanylate kinaselike domain of the PSD-95/SAP90 family of channel clustering molecules. J. Cell Biol. 136, 669-678. doi: 10.1083/jcb.136.3.669

Landis, D. M., and Reese, T. S. (1983). Cytoplasmic organization in cerebellar dendritic spines. J. Cell Biol. 97, 1169-1178. doi: 10.1083/jcb.97.4.1169

Lisman, J., Yasuda, R., and Raghavachari, S. (2012). Mechanisms of CaMKII action in long-term potentiation. Nat. Rev. Neurosci. 13, 169-182. doi: 10. $1038 /$ nrn3192

Lowenthal, M. S., Markey, S. P., and Dosemeci, A. (2015). Quantitative mass spectrometry measurements reveal stoichiometry of principal postsynaptic density proteins. J. Proteome Res. 14, 2528-2538. doi: 10.1021/acs.jproteome. 5 b00109
Martone, M. E., Jones, Y. Z., Young, S. J., Ellisman, M. H., Zivin, J. A., and Hu, B. R. (1999). Modification of postsynaptic densities after transient cerebral ischemia: a quantitative and three-dimensional ultrastructural study. J. Neurosci. 19, 1988-1997.

Naisbitt, S., Kim, E., Tu, J. C., Xiao, B., Sala, C., Valtschanoff, J., et al. (1999). Shank, a novel family of postsynaptic density proteins that binds to the NMDA receptor/PSD-95/GKAP complex and cortactin. Neuron 23, 569-582. doi: 10. 1016/s0896-6273(00)80809-0

Nathan, J. A., Kim, H. T., Ting, L., Gygi, S. P., and Goldberg, A. L. (2013). Why do cellular proteins linked to K63-polyubiquitin chains not associate with proteasomes? EMBO J. 32, 552-565. doi: 10.1038/emboj.2012.354

Okamoto, K., Narayanan, R., Lee, S. H., Murata, K., and Hayashi, Y. (2007). The role of CaMKII as an F-actin-bundling protein crucial for maintenance of dendritic spine structure. Proc. Natl. Acad. Sci. U S A 104, 6418-6423. doi: 10. 1073/pnas.0701656104

Otmakhov, N., Tao-Cheng, J. H., Carpenter, S., Asrican, B., Dosemeci, A., Reese, T. S., et al. (2004). Persistent accumulation of calcium/calmodulindependent protein kinase II in dendritic spines after induction of NMDA receptor-dependent chemical long-term potentiation. J. Neurosci. 24, 9324-9331. doi: 10.1523/JNEUROSCI.2350-04.2004

Palay, S. L. (1956). Synapses in the central nervous system. J. Biophys. Biochem. Cytol. 2, 193-202. doi: 10.1083/jcb.2.4.193

Petersen, J. D., Chen, X., Vinade, L., Dosemeci, A., Lisman, J. E., and Reese, T. S. (2003). Distribution of postsynaptic density (PSD)-95 and $\mathrm{Ca}^{2+} /$ calmodulindependent protein kinase II at the PSD. J. Neurosci. 23, 11270-11278.

Petralia, R. S., Sans, N., Wang, Y. X., and Wenthold, R. J. (2005). Ontogeny of postsynaptic density proteins at glutamatergic synapses. Mol. Cell. Neurosci. 29, 436-452. doi: 10.1016/j.mcn.2005.03.013

Qualmann, B., Boeckers, T. M., Jeromin, M., Gundelfinger, E. D., and Kessels, M. M. (2004). Linkage of the actin cytoskeleton to the postsynaptic density via direct interactions of Abp1 with the ProSAP/Shank family. J. Neurosci. 24, 2481-2495. doi: 10.1523/JNEUROSCI.5479-03.2004

Racz, B., and Weinberg, R. J. (2004). The subcellular organization of cortactin in hippocampus. J. Neurosci. 24, 10310-10317. doi: 10.1523/JNEUROSCI.208004.2004

Rostaing, P., Real, E., Siksou, L., Lechaire, J. P., Boudier, T., Boeckers, T. M., et al. (2006). Analysis of synaptic ultrastructure without fixative using high-pressure freezing and tomography. Eur. J. Neurosci. 24, 3463-3474. doi: 10.1111/j.14609568.2006.05234.x

Rumbaugh, G., Adams, J. P., Kim, J. H., and Huganir, R. L. (2006). SynGAP regulates synaptic strength and mitogen-activated protein kinases in cultured neurons. Proc. Natl. Acad. Sci. U S A 103, 4344-4351. doi: 10.1073/pnas. 0600084103

Sala, C., Piëch, V., Wilson, N. R., Passafaro, M., Liu, G., and Sheng, M. (2001). Regulation of dendritic spine morphology and synaptic function by Shank and Homer. Neuron 31, 115-130. doi: 10.1016/s0896-6273(01)00339-7

Sala, C., Vicidomini, C., Bigi, I., Mossa, A., and Verpelli, C. (2015). Shank synaptic scaffold proteins: keys to understanding the pathogenesis of autism and other synaptic disorders. J. Neurochem. 135, 849-858. doi: 10.1111/jnc. 13232

Schnell, E., Sizemore, M., Karimzadegan, S., Chen, L., Bredt, D. S., and Nicoll, R. A. (2002). Direct interactions between PSD-95 and stargazin control synaptic AMPA receptor number. Proc. Natl. Acad. Sci. U S A 99, 13902-13907. doi: 10. 1073/pnas.172511199

Scita, G., Confalonieri, S., Lappalainen, P., and Suetsugu, S. (2008). IRSp53: crossing the road of membrane and actin dynamics in the formation of membrane protrusions. Trends Cell Biol. 18, 52-60. doi: 10.1016/j.tcb.2007. 12.002

Shonesy, B. C., Jalan-Sakrikar, N., Cavener, V. S., and Colbran, R. J. (2014). CaMKII: a molecular substrate for synaptic plasticity and memory. Prog. Mol. Biol. Transl. Sci. 122, 61-87. doi: 10.1016/B978-0-12-420170-5. 00003-9

Sugiyama, Y., Kawabata, I., Sobue, K., and Okabe, S. (2005). Determination of absolute protein numbers in single synapses by a GFP-based calibration technique. Nat. Methods 2, 677-684. doi: 10.1038/nmeth783

Suzuki, T., Okumura-Noji, K., Tanaka, R., and Tada, T. (1994). Rapid translocation of cytosolic $\mathrm{Ca}^{2+} /$ calmodulin-dependent protein kinase II into postsynaptic density after decapitation. J. Neurochem. 63, 1529-1537. doi: 10. 1046/j.1471-4159.1994.63041529.x 
Tao-Cheng, J. H., Dosemeci, A., Gallant, P. E., Smith, C., and Reese, T. (2010). Activity induced changes in the distribution of Shanks at hippocampal synapses. Neuroscience 168, 11-17. doi: 10.1016/j.neuroscience.2010. 03.041

Tao-Cheng, J. H., Thein, S., Yang, Y., Reese, T. S., and Gallant, P. E. (2014a). Homer is concentrated at the postsynaptic density and does not redistribute after acute synaptic stimulation. Neuroscience 266, 80-90. doi: 10.1016/j. neuroscience.2014.01.066

Tao-Cheng, J. H., Yang, Y., Bayer, K. U., Reese, T. S., and Dosemeci, A. (2014b). NMDA-induced accumulation of Shank at the postsynaptic density is mediated by CaMKII. Biochem. Biophys. Res. Commun. 450, 808-811. doi: 10.1016/j.bbrc. 2014.06.049

Tao-Cheng, J. H., Yang, Y., Reese, T. S., and Dosemeci, A. (2015). Differential distribution of Shank and GKAP at the postsynaptic density. PLoS One 10:e0118750. doi: 10.1371/journal.pone.0118750

Thein, S., Tao-Cheng, J. H., Li, Y., Bayer, K. U., Reese, T. S., and Dosemeci, A. (2014). CaMKII mediates recruitment and activation of the deubiquitinase CYLD at the postsynaptic density. PLoS One 9:e91312. doi: 10.1371/journal. pone.0091312

Tu, J. C., Xiao, B., Naisbitt, S., Yuan, J. P., Petralia, R. S., Brakeman, P., et al. (1999). Coupling of mGluR/Homer and PSD-95 complexes by the Shank family of postsynaptic density proteins. Neuron 23, 583-592. doi: 10.1016/s08966273(00)80810-7

Valtschanoff, J. G., and Weinberg, R. J. (2001). Laminar organization of the NMDA receptor complex within the postsynaptic density. J. Neurosci. 21, 1211-1217.

Walikonis, R. S., Oguni, A., Khorosheva, E. M., Jeng, C. J., Asuncion, F. J., and Kennedy, M. B. (2001). Densin-180 forms a ternary complex with the $\alpha$-subunit of $\mathrm{Ca}^{2+} /$ calmodulin-dependent protein kinase II and $\alpha$-actinin. J. Neurosci. 21, $423-433$.

Xiao, B., Tu, J. C., Petralia, R. S., Yuan, J. P., Doan, A., Breder, C. D., et al. (1998). Homer regulates the association of group 1 metabotropic glutamate receptors with multivalent complexes of homer-related, synaptic proteins. Neuron 21, 707-716. doi: 10.1016/s0896-6273(00)80588-7

Yang, Y., Tao-Cheng, J. H., Bayer, K. U., Reese, T. S., and Dosemeci, A. (2013). Camkii-mediated phosphorylation regulates distributions of Syngap- $\alpha 1$ and $-\alpha 2$ at the postsynaptic density. PLoS One 8:e71795. doi: 10.1371/journal. pone.0071795

Yang, Y., Tao-Cheng, J. H., Reese, T. S., and Dosemeci, A. (2011). SynGAP moves out of the core of the postsynaptic density upon depolarization. Neuroscience 192, 132-139. doi: 10.1016/j.neuroscience.2011.06.061

Zhao, H., Pykalainen, A., and Lappalainen, P. (2011). I-BAR domain proteins: linking actin and plasma membrane dynamics. Curr. Opin. Cell Biol. 23, 14-21. doi: 10.1016/j.ceb.2010.10.005

Conflict of Interest Statement: The authors declare that the research was conducted in the absence of any commercial or financial relationships that could be construed as a potential conflict of interest.

Copyright (C) 2016 Dosemeci, Weinberg, Reese and Tao-Cheng. This is an open-access article distributed under the terms of the Creative Commons Attribution License (CC BY). The use, distribution and reproduction in other forums is permitted, provided the original author(s) or licensor are credited and that the original publication in this journal is cited, in accordance with accepted academic practice. No use, distribution or reproduction is permitted which does not comply with these terms. 